\title{
Quantum Markov Order
}

\author{
Philip Taranto, ${ }^{1, *}$ Felix A. Pollock, ${ }^{1}$ Simon Milz, ${ }^{1}$ Marco Tomamichel, ${ }^{2}$ and Kavan Modi ${ }^{1}$ \\ ${ }^{1}$ School of Physics \& Astronomy, Monash University, Clayton, Victoria 3800, Australia \\ ${ }^{2}$ Centre for Quantum Software and Information, School of Software, University of Technology Sydney, Sydney NSW 2007, Australia
}

(Received 9 November 2018; published 9 April 2019)

\begin{abstract}
We formally extend the notion of Markov order to open quantum processes by accounting for the instruments used to probe the system of interest at different times. Our description recovers the classical property in the appropriate limit: when the stochastic process is classical and the instruments are noninvasive, i.e., restricted to orthogonal, projective measurements. We then prove that there do not exist non-Markovian quantum processes that have finite Markov order with respect to all possible instruments; the same process exhibits distinct memory effects when probed by different instruments. This naturally leads to a relaxed definition of quantum Markov order with respect to specified instrument sequences. The memory effects captured by different choices of instruments vary dramatically, providing a rich landscape for future exploration.
\end{abstract}

DOI: 10.1103/PhysRevLett.122.140401

Introduction.-Fundamentally, physical laws are local in time, yet memory effects pervade processes studied throughout the sciences, since no system is isolated [1]. Our inability to capture interactions between a system of interest and its environment leads to stochastic dynamics for the system, with information about its history influencing future evolution, often leading to a buildup of correlations over time [2]. Such temporal correlations are exhibited over various timescales in complex phenomena. However, a natural notion of memory length emerges in the context of statistical modeling: the amount of a system's history that directly affects its future. This, importantly, dictates the resources required for simulation, which grow exponentially in the memory length (even classically) [3-5]. Fortunately, most processes have an effectively finite-length memory, permitting an efficient description that considers only the portion of history necessary to predict the future [6]. Alternatively, given control over some quantum degrees of freedom for some duration, any process with the corresponding memory length can be simulated. Indeed, manipulating memory effects has proven advantageous in various information-processing tasks such as preserving coherence [7-11]. Clearly, memory will need to be exploited to develop near-term quantum technologies.

In the classical setting, the finite-length memory approximation underpins the often-invoked order- $\ell$ Markov models, which use information of only the past $\ell$ observed states to predict the next. However, even in the simplest case of memoryless, or Markovian, dynamics (i.e., $\ell=1$ ), the study of stochastic processes is vastly different in quantum mechanics than its classical counterpart, mainly because, in the former, one must necessarily disturb the system to observe realizations of the process, breaking an implicit classical assumption [12-15]. Crucially, this leads to a breakdown of the Kolmogorov extension theorem [16-18], which provides the mathematical foundation of stochastic processes that allows for calculation of conditional probability distributions. Conventional approaches to quantum stochastic processes attempt to sidestep this problem by describing properties of the process in terms of the time-evolving system density operator, failing to capture multitime effects [19]; others constrain systemenvironment interactions to specify memory mechanisms [20-22]; both perspectives lead to necessary but insufficient criteria for Markovianity [23].

The aforementioned issues can be circumvented by separating the controllable influence on the system from the underlying process, as achieved by various modern frameworks, including the process matrix [24] and process tensor formalisms $[25,26]$. These represent processes as quantum combs [27-29], mapping sequences of probing instruments to accessible joint probability distributions through a generalized spatiotemporal Born rule [30]. They have been used to extend the causal modeling paradigm (originally developed for classical processes [31]) to quantum theory [32-36]. Most importantly for our purposes, by capturing all multitime correlations, these frameworks provide unambiguous conditions for a process to be Markovian, unifying all previous approaches [26]. Like the joint probability distribution characterizing classical stochastic processes, a quantum stochastic process suffers exponentially increasing complexity with respect to its memory length, with the added complication that all possible sequences of interventions must be accounted for. This naturally begs the question: are there quantum processes with finite-length memory, and hence significantly reduced complexity? 
In this Letter, we extend the notion of Markov order to quantum stochastic processes. We begin by discussing classical Markov order to motivate its generalization to the quantum realm. We use the process tensor formalism to prove our main result: non-Markovian quantum processes, generically, have infinite Markov order. Afterwards, we formulate the conditions for a quantum process to have finite Markov order in a constrained setting. The structure of quantum Markov order is far richer than its classical counterpart, as explored in detail in an accompanying article [37].

Classical Markov order.-The concept of Markov order is essentialized by the following question: is knowledge of a portion of the history of a process sufficient to predict future statistics? Consider an $(n+1)$-step classical stochastic process, segmented into three intervals: the future $F=\left\{t_{n}, \ldots, t_{k}\right\}$, the memory $M=\left\{t_{k-1}, \ldots, t_{k-\ell}\right\}$, and the history $H=\left\{t_{k-\ell-1}, \ldots, t_{0}\right\}$ (in principle, the history and future can extend infinitely long). The random variables $X_{j}$ describing the system (with the subscript denoting the time step) are grouped similarly: $\left\{X_{F}, X_{M}, X_{H}\right\}$. The Markov order of the process is defined in terms of the conditional statistics of these random variables:

Definition 1.-(classical Markov order) A classical stochastic process has Markov order $\ell$ if the conditional probability for any realization $x_{F}$ of the random variables $X_{F}$ beyond any time $0<t_{k}<t_{n}$ depends only on the realizations $x_{M}$ of those in the previous $\ell$ time steps, and not on realizations $x_{H}$ of the earlier history:

$$
\mathbb{P}_{F}\left(x_{F} \mid x_{M}, x_{H}\right)=\mathbb{P}_{F}\left(x_{F} \mid x_{M}\right) .
$$

As a special case, $\ell=1$ corresponds to a Markovian process.

The property of Markov order $\ell$ constrains the underlying joint probability distribution characterizing the process, from which these conditional distributions arise. It follows from Eq. (1) that, for any realization of events in any length $\ell$ block $M$, the joint conditional distribution over $F$ and $H$ factorizes,

$$
\mathbb{P}_{F H}\left(x_{F}, x_{H} \mid x_{M}\right)=\mathbb{P}_{F}\left(x_{F} \mid x_{M}\right) \mathbb{P}_{H}\left(x_{H} \mid x_{M}\right) .
$$

In other words, the future and the history are conditionally independent, given specification of events in the memory. This conditional independence is equivalently expressed by the vanishing classical conditional mutual information (CMI), $I(F: H \mid M)=0$.

While Markov order $\ell$ dictates that the next state depends only upon the previous $\ell$, it does not imply an absolute demarcation of time steps into blocks of memory and irrelevant history. Instead, the memory blocks corresponding to different time steps overlap, permitting the existence of unconditional correlations between time steps with separation greater than $\ell$ in general [38]; however,

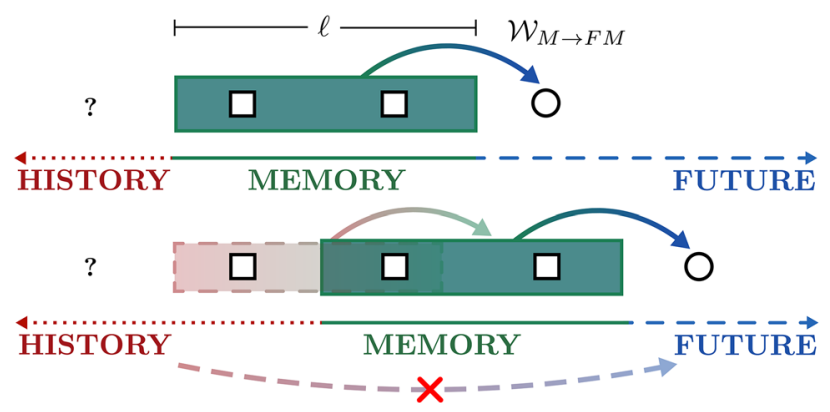

FIG. 1. Knowledge of the $\ell=2$ states in the memory block suffices to predict the future statistics via the recovery map $\mathcal{W}_{M \rightarrow F M}$. No information about the history is required to determine these probabilities, indicated by the question mark. This property holds true for all time steps. Any influence the history (beyond $\ell$ time steps ago) has on the future is mediated through memory blocks; conditional on the states in the most recent block, there are no correlations between history and future, indicated by the dashed arrow.

such correlations are always mediated through overlapping memory blocks (see Fig. 1). Markov order thus quantifies how much of the history one must remember to predict the future, providing a natural "measure" for the memory length of the process.

Operationally, the significance of finite memory length is best encapsulated through the notion of a recovery map, $\mathcal{W}_{M \rightarrow F M}$, which acts only on $M$ to give the correct future statistics: $\quad \mathbb{P}_{F M H}\left(x_{F}, x_{M}, x_{H}\right)=\mathcal{W}_{M \rightarrow F M}\left[\mathbb{P}_{M H}\left(x_{M}, x_{H}\right)\right]$. The complexity of any predictive model is fundamentally bounded by the length of the block $M$ on which it acts (as well as the number of possible realizations of each $X_{j}$ ). Recently, the recovery map has featured in the quantum information literature: here, quantum Markov chains are defined as states with vanishing quantum CMI $[39,40]$, or, equivalently, those satisfying quantum generalizations of recoverability [41-46]. However, it is unclear how such characterizations relate to temporal processes, where one has access to an evolving quantum system across multiple times. We now consider a framework that provides the most general description of quantum (and classical) stochastic processes, allowing us to extend the concept of Markov order to quantum processes.

Quantum stochastic processes.-A classical stochastic process is characterized by the joint probability distribution $\mathbb{P}\left(x_{n}, t_{n} ; \ldots ; x_{2}, t_{2} ; x_{1}, t_{1}\right)$ over events at different times, expressing, e.g., the probability for a molecule to be found in region $x_{1}$ at time $t_{1}$ and region $x_{2}$ at $t_{2}$, and so forth. Analogously, a quantum stochastic process can be considered as a set of joint probability distributions for a sequence of measurement outcomes. However, in contrast, there is a continuous family of possible measurements and the choice of measurement at one time (or even whether to measure at all) can affect future statistics [18,25,26]. To account for this, we separate the controllable influence applied to the 
system corresponding to a set of measurement outcomes $\{x\}$ given a choice of experimental instrument $\mathcal{J}$ from the uncontrollable underlying process.

On the controllable side, the experimenter can apply any valid transformation to the system at each time step, formally described by an instrument. Mathematically, an instrument is a collection of completely positive (CP) maps $\mathcal{J}_{j}=\left\{O_{j}^{\left(x_{j}\right)}\right\}$ that chronicle the transformation the system undergoes upon realization of each measurement outcome. Specifying an instrument at time step $t_{j}$ allows an experimenter to observe outcome $x_{j}$ with probability $\mathbb{P}_{j}\left(x_{j} \mid \mathcal{J}_{j}\right)$. The average transformation effected by the instrument is given by the completely positive trace-preserving (CPTP) map $O_{j}^{\mathcal{J}_{j}}:=\sum_{x_{j}} O_{j}^{\left(x_{j}\right)}$. Without loss of generality, we use the Choi-Jamiołkowski isomorphism to represent all such maps as bipartite quantum states [15,47,48]. Moreover, instruments can be extended across multiple time steps, describing correlated measurements and repeated interactions with an ancilla; implementing such an instrument sequence yields the joint statistics $\mathbb{P}_{n: 0}\left(x_{n: 0} \mid \mathcal{J}_{n: 0}\right)$. The corresponding correlated transformations to the system associated to observing a sequence $x_{n: 0}$ can be represented as a many-body Choi state $O_{n: 0}^{\left(x_{n}\right)}$.

Crucially, to describe quantum stochastic processes, one must distinguish between such instruments and the underlying process. The former constitutes all that is controllable by an experimenter, while the latter stems from the uncontrollable system-environment dynamics. The process itself is encapsulated in the process tensor, $\Upsilon_{n: 0}$, whose Choi state is a multipartite density operator, naturally generalizing the joint probability distributions that characterize classical stochastic processes [49]. The process tensor is a linear map taking any sequence of transformations $O_{n: 0}^{\left(x_{n: 0}\right)}$ to the corresponding joint probability distribution of its realization; it thus contains all multitime probabilities deducible by all possible instrument sequences, calculated via

$$
\mathbb{P}_{n: 0}\left(x_{n: 0} \mid \mathcal{J}_{n: 0}\right)=\operatorname{tr}\left[O_{n: 0}^{\left(x_{n: 0}\right)} \Upsilon_{n: 0}\right]
$$

This is a temporal generalization of the Born rule [30], and is directly analogous to its spatial counterpart, which relates observed statistics to measurement operators (instead of instruments) and a density operator (instead of a process tensor). That such an object exists is a consequence of the linearity of quantum mechanics; like the density operator, the process tensor can be (and has been [50]) tomographically constructed in a finite number of experiments. In anticipation of our main results, we emphasize that Eq. (3) can be used to deduce conditional processes given specification of outcomes over a subset of time steps by restricting the trace appropriately (see Appendix A in Supplemental Material [51]).
The process tensor extends the CPTP map paradigm to capture multitime effects, and can be simulated using open systems techniques capable of computing multitime correlations [25]. Once known, it allows one to, e.g., calculate the system density operator at each time step, but, importantly, also includes all multitime correlations, providing the most general description of open dynamics within quantum and classical physics. Notably, this approach has been used to prove that quantum processes satisfy a generalized Kolmogorov extension theorem [18], thereby allowing joint and conditional probability distributions to be calculated. Crucially, we can now meaningfully construct quantum generalizations of Definition 1, granting a fundamental study of memory in quantum processes.

Quantum Markov order.-For any fixed choice of instruments used to probe a quantum process, one yields a probability distribution describing a classical stochastic process [26]. A natural approach to extending Markov order to quantum processes is to demand such classical processes satisfy Definition 1; however, each choice of instruments generally leads to statistics describing different classical processes. Nonetheless, a sensible requirement of a quantum process with finite-length memory is that any future statistics deducible (no matter which future instruments are chosen) are conditionally independent of any historical statistics, given knowledge of a length- $\ell$ instrument sequence on the memory. We define quantum Markov order accordingly:

Definition 2.-(quantum Markov order) A quantum stochastic process has Markov order $\ell$ with respect to a family of instruments $\left\{\mathcal{J}_{M}\right\}$ when the statistics deducible from the process satisfy Definition 1:

$\mathbb{P}_{F}\left(x_{F} \mid \mathcal{J}_{F} ; x_{M}, \mathcal{J}_{M} ; x_{H}, \mathcal{J}_{H}\right)=\mathbb{P}_{F}\left(x_{F} \mid \mathcal{J}_{F} ; x_{M}, \mathcal{J}_{M}\right)$,

for each $\mathcal{J}_{M}$ and for all possible history and future instruments $\mathcal{J}_{H}$ and $\mathcal{J}_{F}$.

Intuitively, this means that for any future instruments one might apply to the system, the statistics of different measurement outcomes are determined by the most recent $\ell$ instruments and outcomes. Equivalently, given specification of the outcomes of the past $\ell$ instruments, the process governing the future dynamics is uncorrelated with that of the history, guaranteeing that any deducible statistics on the history and future are independent. Definition 2 leads to the following product structure condition on the process tensor (see Appendix B in Supplemental Material [51]):

$$
\Upsilon_{F H}^{\left(x_{M}\right)}:=\operatorname{tr}_{M}\left[O_{M}^{\left(x_{M}\right)} \Upsilon_{F M H}\right]=\Upsilon_{F}^{\left(x_{M}\right)} \otimes \Upsilon_{H}^{\left(x_{M}\right)}
$$

for all $O_{M}^{\left(x_{M}\right)} \in \mathcal{J}_{M}$. In analogy to Eq. (2), the conditional history and future processes are independent for each realization of the instrument applied. Importantly, Definition 2 reduces to Definition 1 in the correct limit: 
Theorem 3: When restricted to classical stochastic processes, Definition 2 reduces to Definition 1 for any choice of (sharp) classical instruments.

Proof.-It suffices to show that when restricted to probing a classical stochastic process with sharp classical instruments, $\mathbb{P}_{F M H}\left(x_{F}, x_{M}, x_{H} \mid \mathcal{J}_{F}^{\mathrm{cl}}, \mathcal{J}_{M}^{\mathrm{cl}}, \mathcal{J}_{H}^{\mathrm{cl}}\right)=\mathbb{P}_{F M H}\left(x_{F}, x_{M}, x_{H}\right)$. Sharp classical instruments correspond to a complete set of (rank-1) projections onto orthogonal states at each time step: $\mathcal{J}^{\mathrm{cl}}=\left\{\Pi_{i}^{(x)} \otimes \Pi_{\circ}^{(x)}\right\}$, where $\Pi_{i}^{(x)}=\Pi_{0}^{(x)}:=|x\rangle\langle x|$ satisfy $\operatorname{tr}\left[\Pi^{(x)} \Pi^{(y)}\right]=\delta_{x y} \forall x, y$ (i and $\circ$ refer, respectively, to the input and output spaces of the maps applied at each time step). A stochastic process arising from classical physics, i.e., a joint probability distribution, can be encoded in the diagonal of a process tensor with respect to the local product basis that the measurements act in; thus, the process tensor has the following structure: $\Upsilon_{F M H}^{\mathrm{cl}}=\sum \mathbb{P}_{F M H}\left(y_{F}, y_{M}, y_{H}\right) \Pi_{F^{\mathrm{i}}}^{\left(y_{F}\right)} \otimes \Pi_{M^{\mathrm{i}}}^{\left(y_{M}\right)} \otimes \Pi_{H^{\mathrm{i}}}^{\left(y_{H}\right)} \otimes \mathbb{1}_{F M H}^{\circ}$. Evaluating $\mathbb{P}_{F M H}\left(x_{F}, x_{M}, x_{H} \mid \mathcal{J}_{F}^{\mathrm{cl}}, \mathcal{J}_{M}^{\mathrm{cl}}, \mathcal{J}_{H}^{\mathrm{cl}}\right)$ according to Eq. (3) yields $\mathbb{P}_{F M H}\left(x_{F}, x_{M}, x_{H}\right)$.

Thus, our definition generalizes Markov order to quantum mechanics. However, for quantum processes, a much richer arsenal of instruments can be implemented. Demanding that Eq. (4) holds for arbitrary instrument sequences trivializes the theory:

Theorem 4: The only quantum processes with finite Markov order with respect to all possible instruments are Markovian.

The proof uses the fact that the set of $\mathrm{CP}$ maps forms a vector space to show that the only processes satisfying Eq. (5) for all instruments have trivial Markov order (see Appendix C in Supplemental Material [51]). Specifically, we show that if a process has finite Markov order for a complete basis of $\mathrm{CP}$ maps on $M$, it cannot have finite Markov order with respect to any linear combination of them. This implies the following property:

Remark.-Any non-Markovian quantum process has infinite Markov order with respect to a generic instrument sequence.

In light of this finding, it is clear that the classical Markov order statement in Definition 1 is weak, as it does not consider how one measures outcomes and assumes the ability for sharp observations. Indeed, when one allows for noisy classical measurements, the product structure of Eq. (2) breaks down, even for Markovian processes [52]. This is not due to intrinsic memory of the process; rather, due to information about the history leaking into the future, thanks to the fuzziness of the measurements. Although this issue is liftable in classical physics, in quantum mechanics it is fundamental: even sharp quantum measurements appear noisy as they do not generally reveal the full state of the system. In contrast to the classical statement, demanding Definition 2 to hold for all instruments is strong, requiring the observed statistics to satisfy the Markov order- $\ell$ property no matter how they are measured;

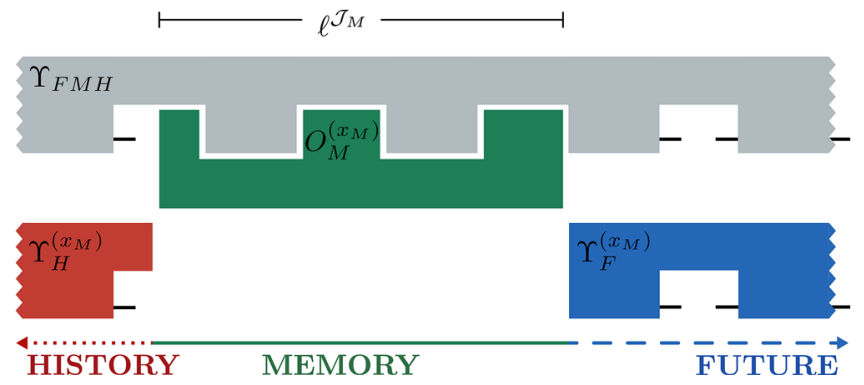

FIG. 2. An instrument $\mathcal{J}_{M}$, comprising (temporally correlated) CP maps $\left\{O_{M}^{\left(x_{M}\right)}\right\}$ (green) over $\ell$ time steps, is applied to a process $\Upsilon_{F M H}$. The process has Markov order $\ell$ with respect to this instrument, denoted $\ell^{\mathcal{J}_{M}}$ when, for each possible realization $x_{M}$, the history (red) and future (blue) conditional processes are independent.

Theorem 4 shows that this is too restrictive. This result motivates the following introduction of a relaxed, instrument-specific definition for quantum Markov order.

Instrument-specific quantum Markov order.-We say that a stochastic process has quantum Markov order $\ell$ with respect to the instrument sequence $\mathcal{J}_{M}$ when Definition 2 is satisfied for each realization of the sequence in question. In terms of the process tensor, this implies that there exists an instrument such that Eq. (5) is satisfied. Importantly, while the instrument on the memory block must be specified, the history and future instruments remain arbitrary: for each realization of the memory instrument, any deducible statistics on the history and future are conditionally independent. This is illustrated in Fig. 2, where the transformations $O_{M}^{\left(x_{M}\right)}$ that "break apart" the process are temporally correlated (as they will be generically). Interestingly, quantum processes with finite Markov order have starkly distinct properties from their classical counterparts.

Proposition 5: In contrast to classical processes, quantum processes with finite instrument-specific quantum Markov order can have nonvanishing quantum CMI.

Proof.-Consider $\Upsilon_{F M H}=\sum_{x} \mathbb{P}(x) \Upsilon_{F}^{(x)} \otimes \Delta_{M}^{(x)} \otimes \Upsilon_{H}^{(x)}$ such that $\Upsilon_{F M H} \geq 0$ and $\operatorname{tr}\left[\Delta_{M}^{(x)} O_{M}^{(y)}\right]=\delta_{x y} \forall x, y$, where each $O_{M}^{(y)}$ is an element of some instrument $\mathcal{J}_{M}=\left\{O_{M}^{(y)}\right\}$. Such processes can have nonvanishing quantum CMI, $I(F: H \mid M)>0$ when the Choi states of the $O_{M}^{(y)}$ do not all commute; indeed, $I(F: H \mid M)$ is not monotonic with respect to instruments in $M$, and is therefore a poor quantifier for memory strength. Nonetheless, such processes have finite Markov order with respect to the instrument $\mathcal{J}_{M}$ (see Appendix D in Supplemental Material [51]).

As highlighted above, quantum Markov order permits a vast landscape of memory effects: (i) the decoupling instruments can vary across time steps (or even be necessarily correlated); (ii) at each time step, instruments need not comprise only orthogonal projectors; (iii) deterministic 
instruments can break future-history correlations; and (iv) quantum CMI is not necessarily vanishing for processes with finite quantum Markov order. In an accompanying article [37], we explore the structure of processes with finite quantum Markov order with respect to natural classes of instruments, shedding light on such distinguishing features. We now discuss the broader implications of our work.

Conclusions.-In this Letter we have formulated an extension of Markov order to the quantum realm, which reduces to the classical condition appropriately. Theorem 4 shows that demanding the proposed condition to hold for all possible instruments is too strict, immediately trivializing the theory. The implication is that, generically, nonMarkovian quantum processes have infinite Markov order; they exhibit distinct memory effects when probed differently. Interestingly, such instrument-specific effects have been observed [53]; our characterization formally explains such behavior.

This led us to propose a relaxed definition of instrumentspecific quantum Markov order. Here, the history and future processes are independent conditioned on each outcome of an instrument sequence specified on the memory. Perhaps surprisingly, when one allows for noisy measurements (or interventions more generally) in the classical setting, a similar relaxation is necessary. This has significant implications for the reconstruction of complex dynamics [52,54-56], highlighting that the standard formulation of Markov order is weaker than is necessary in quantum mechanics, where measurements are inherently fuzzy.

Our framework opens the door for a comprehensive and unambiguous study of memory effects in quantum processes, which has hitherto been elusive. By capturing multitime statistics, it goes beyond state-of-the-art descriptions which typically only consider two-point correlations and thus cannot properly describe memory effects.

Non-Markovian processes with strictly finite Markov order are unlikely to be found in nature; however, numerical techniques for open dynamics often invoke finite memory approximations, where rapidly vanishing temporal correlations are truncated [57-59]. This is tantamount to treating the process as having finite Markov order with respect to the identity instrument (i.e., do nothing) when the correlations considered involve operators evolved freely by the underlying Hamiltonian, although memory approximations involving other choices of instruments can also be made [60].

Understanding memory effects has immediate relevance to developing near-term quantum technologies, particularly concerning the construction of error-correcting codes to combat correlated noise [61-65] and the design of feedback protocols for coherent control $[66,67]$. Our work poses the following questions for further investigation: Which instrument sequence is optimal in blocking the influence of a given process's history? What constraints are imposed on the underlying system-environment dynamics? How can we measure memory strength to characterize processes with approximately finite memory, and what are the subsequent implications for recoverability? These questions, among others, are critical for both our foundational understanding of quantum theory and the efficient simulation of quantum processes.

We thank Top Notoh for insightful discussions. P. T. is supported by the Australian Government Research Training Program Scholarship and the J. L. William Scholarship. S. M. is supported by the Monash Graduate Scholarship, the Monash International Postgraduate Research Scholarship, and the J.L. William Scholarship. K. M. is supported through Australian Research Council Future Fellowship FT160100073.

*philip.taranto@monash.edu

[1] N. Van Kampen, Stochastic Processes in Physics and Chemistry (Elsevier, New York, 2007).

[2] H.-P. Breuer and F. Petruccione, The Theory of Open Quantum Systems (Oxford University Press, New York, 2002).

[3] S. L. Salzberg, A. L. Delcher, S. Kasif, and O. White, Nucleic Acids Res. 26, 544 (1998).

[4] G. Thijs, M. Lescot, K. Marchal, S. Rombauts, B. De Moor, P. Rouze, and Y. Moreau, Bioinformatics 17, 1113 (2001).

[5] M. Rosvall, A. V. Esquivel, A. Lancichinetti, J. D. West, and R. Lambiotte, Nat. Commun. 5, 4630 (2014).

[6] F. A. Pollock and K. Modi, Quantum 2, 76 (2018).

[7] M. Nielsen and I. Chuang, Quantum Computation and Quantum Information (Cambridge University Press, Cambridge, England, 2000).

[8] L. Viola, E. Knill, and S. Lloyd, Phys. Rev. Lett. 82, 2417 (1999).

[9] K. Banaszek, A. Dragan, W. Wasilewski, and C. Radzewicz, Phys. Rev. Lett. 92, 257901 (2004).

[10] N. Erez, G. Gordon, M. Nest, and G. Kurizki, Nature (London) 452, 724 (2008).

[11] D. M. Reich, N. Katz, and C. P. Koch, Sci. Rep. 5, 12430 (2015).

[12] K. Modi, Open Syst. Inf. Dyn. 18, 253 (2011).

[13] K. Modi, Sci. Rep. 2, 581 (2012).

[14] K. Modi, C. A. Rodríguez-Rosario, and A. Aspuru-Guzik, Phys. Rev. A 86, 064102 (2012).

[15] S. Milz, F. A. Pollock, and K. Modi, Open Syst. Inf. Dyn. 24, 1740016 (2017).

[16] A. N. Kolmogorov, Foundations of the Theory of Probability [Grundbegriffe der Wahrscheinlichkeitsrechnung] (Chelsea, New York, 1956).

[17] W. Feller, An Introduction to Probability Theory and its Applications (Wiley, New York, 1971).

[18] S. Milz, F. Sakuldee, F. A. Pollock, and K. Modi, arXiv:1712.02589.

[19] H.-P. Breuer, E.-M. Laine, J. Piilo, and B. Vacchini, Rev. Mod. Phys. 88, 021002 (2016). 
[20] V. Giovannetti and G. M. Palma, Phys. Rev. Lett. 108, 040401 (2012).

[21] S. Lorenzo, F. Ciccarello, and G. M. Palma, Phys. Rev. A 96, 032107 (2017).

[22] S. Lorenzo, F. Ciccarello, G. M. Palma, and B. Vacchini, Open Syst. Inf. Dyn. 24, 1740011 (2017).

[23] L. Li, M. J. Hall, and H. M. Wiseman, Phys. Rep., 7591 (2018).

[24] O. Oreshkov, F. Costa, and Č. Brukner, Nat. Commun. 3, 1092 (2012).

[25] F. A. Pollock, C. Rodríguez-Rosario, T. Frauenheim, M. Paternostro, and K. Modi, Phys. Rev. A 97, 012127 (2018).

[26] F. A. Pollock, C. Rodríguez-Rosario, T. Frauenheim, M. Paternostro, and K. Modi, Phys. Rev. Lett. 120, 040405 (2018).

[27] G. Chiribella, G. M. D’Ariano, and P. Perinotti, Europhys. Lett. 83, 30004 (2008).

[28] G. Chiribella, G. M. D’Ariano, and P. Perinotti, Phys. Rev. Lett. 101, 060401 (2008).

[29] G. Chiribella, G. M. D’Ariano, and P. Perinotti, Phys. Rev. A 80, 022339 (2009).

[30] S. Shrapnel, F. Costa, and G. Milburn, New J. Phys. 20 053010 (2018).

[31] J. Pearl, Causality (Oxford University Press, New York, 2000).

[32] M. Araújo, C. Branciard, F. Costa, A. Feix, C. Giarmatzi, and Č. Brukner, New J. Phys. 17, 102001 (2015).

[33] F. Costa and S. Shrapnel, New J. Phys. 18, 063032 (2016).

[34] O. Oreshkov and C. Giarmatzi, New J. Phys. 18, 093020 (2016).

[35] J.-M. A. Allen, J. Barrett, D. C. Horsman, C. M. Lee, and R. W. Spekkens, Phys. Rev. X 7, 031021 (2017).

[36] M. Ringbauer and R. Chaves, Quantum 1, 35 (2017).

[37] P. Taranto, S. Milz, F. A. Pollock, and K. Modi, Phys. Rev. A 98, 042108 (2019).

[38] These are themselves often referred to as memory. Here, we distinguish the total temporal correlations between observables from those resulting from non-Markovian memory, which can survive interventions that reset the system's state.

[39] M. B. Ruskai, J. Math. Phys. 43, 4358 (2002).

[40] D. Petz, Rev. Math. Phys. 15, 79 (2003).

[41] P. Hayden, R. Jozsa, D. Petz, and A. Winter, Commun. Math. Phys. 246, 359 (2004).

[42] B. Ibinson, N. Linden, and A. Winter, Commun. Math. Phys. 277, 289 (2008).
[43] O. Fawzi and R. Renner, Commun. Math. Phys. 340, 575 (2015).

[44] M. M. Wilde, Proc. Royal Soc. A 471, 2182 (2015).

[45] D. Sutter, O. Fawzi, and R. Renner, Proc. R. Soc. A 472, 20150623 (2016).

[46] D. Sutter, M. Berta, and M. Tomamichel, Commun. Math. Phys. 352, 37 (2017).

[47] A. Jamiołkowski, Rep. Math. Phys. 3, 275 (1972).

[48] M.-D. Choi, Linear Algebra Appl. 10, 285 (1975).

[49] S. Milz, F. A. Pollock, T. P. Le, G. Chiribella, and K. Modi, New J. Phys. 20, 033033 (2018).

[50] M. Ringbauer, C. J. Wood, K. Modi, A. Gilchrist, A. G. White, and A. Fedrizzi, Phys. Rev. Lett. 114, 090402 (2015).

[51] See Supplemental Material at http://link.aps.org/ supplemental/10.1103/PhysRevLett.122.140401 for additional details.

[52] D. Kleinhans, R. Friedrich, M. Wächter, and J. Peinke, Phys. Rev. E 76, 041109 (2007).

[53] K. Wiesner and J. P. Crutchfield, arXiv:quant-ph/0611143.

[54] M. Siefert, A. Kittel, R. Friedrich, and J. Peinke, Europhys. Lett. 61, 466 (2003).

[55] F. Böttcher, J. Peinke, D. Kleinhans, R. Friedrich, P. G. Lind, and M. Haase, Phys. Rev. Lett. 97, 090603 (2006).

[56] B. Lehle, Phys. Rev. E 83, 021113 (2011).

[57] N. Makri and D. E. Makarov, J. Chem. Phys. 102, 4600 (1995).

[58] N. Makri and D. E. Makarov, J. Chem. Phys. 102, 4611 (1995).

[59] I. de Vega and D. Alonso, Rev. Mod. Phys. 89, 015001 (2017).

[60] F. A. Pollock and K. Modi, Quantum 2, 76 (2018).

[61] J. P. Clemens, S. Siddiqui, and J. Gea-Banacloche, Phys. Rev. A 69, 062313 (2004).

[62] G. Chiribella, M. Dall'Arno, G. M. D'Ariano, C. Macchiavello, and P. Perinotti, Phys. Rev. A 83, 052305 (2011).

[63] A. Ben-Aroya and A. Ta-Shma, IEEE Trans. Inf. Theory 57, 3982 (2011).

[64] C. Lupo, L. Memarzadeh, and S. Mancini, Phys. Rev. A 85, 012320 (2012).

[65] C. L. Edmunds, C. Hempel, R. Harris, H. Ball, V. Frey, T. M. Stace, and M. J. Biercuk, arXiv:1712.04954.

[66] A. L. Grimsmo, Phys. Rev. Lett. 115, 060402 (2015).

[67] S. J. Whalen, A. L. Grimsmo, and H. J. Carmichael, Quantum Sci. Technol. 2, 044008 (2017). 